\title{
Subthalamic Single Cell and Oscillatory Neural Dynamics of a Dyskinetic Medicated Patient With Parkinson's Disease
}

\author{
Musa Ozturk ${ }^{1}$, Heet Kaku ${ }^{1}$, Joohi Jimenez-Shahed ${ }^{2}$, Ashwin Viswanathan ${ }^{3}$, \\ Sameer A. Sheth ${ }^{3}$, Suneel Kumar ${ }^{4}$ and Nuri F. Ince ${ }^{1 *}$
}

${ }^{1}$ Department of Biomedical Engineering, University of Houston, Houston, TX, United States, ${ }^{2}$ Department of Neurology, Icahn School of Medicine at Mount Sinai, New York, NY, United States, ${ }^{3}$ Department of Neurosurgery, Baylor College of Medicine, Houston, TX, United States, ${ }^{4}$ Department of Neurology, Baylor College of Medicine, Houston, TX, United States

\section{OPEN ACCESS}

Edited by:

Gianfranco Spalletta

Santa Lucia Foundation (IRCCS), Italy

Reviewed by:

Sara Marceglia,

University of Trieste, Italy

Francesco Ernesto Pontieri,

Sapienza University of Rome, Italy

Ron Levy,

Queen's University, Canada

*Correspondence:

Nuri F. Ince

nfince@uh.edu

Specialty section:

This article was submitted to

Neurodegeneration,

a section of the journal

Frontiers in Neuroscience

Received: 18 December 2019 Accepted: 30 March 2020

Published: 24 April 2020

Citation:

Ozturk M, Kaku H, Jimenez-Shahed J,

Viswanathan A, Sheth SA, Kumar S and Ince NF (2020) Subthalamic

Single Cell and Oscillatory Neural

Dynamics of a Dyskinetic Medicated

Patient With Parkinson's Disease.

Front. Neurosci. 14:391.

doi: 10.3389/fnins.2020.00391
Single cell neuronal activity (SUA) and local field potentials (LFP) in the subthalamic nucleus (STN) of unmedicated Parkinson's disease (PD) patients undergoing deep brain stimulation (DBS) surgery have been well-characterized during microelectrode recordings (MER). However, there is limited knowledge about the changes in the firing patterns and oscillations above and within the territories of STN after the intake of dopaminergic medication. Here, for the first time, we report the STN single cell and oscillatory neural dynamics in a medicated patient with idiopathic PD using intraoperative MER. We recorded LFP and SUA with microelectrodes at various depths during bilateral STN-DBS electrode implantation. We isolated 26 neurons in total and observed that tonic and irregular firing patterns of individual neurons predominated throughout the territories of STN. While burst-type firings have been well-characterized in the dorsal territories of STN in unmedicated patients, interestingly, this activity was not observed in our medicated subject. LFP recordings lacked the excessive beta $(8-30 \mathrm{~Hz})$ activity, characteristic of the unmedicated state and signal energy was mainly dominated by slow oscillations below $8 \mathrm{~Hz}$. We observed sharp gamma oscillations between 70 and $90 \mathrm{~Hz}$ within and above the STN. Despite the presence of a broadband high frequency activity in $200-400 \mathrm{~Hz}$ range, no cross-frequency interaction in the form of phase-amplitude coupling was noted between low and high frequency oscillations of LFPs. While our results are in agreement with the previously reported LFP recordings from the DBS lead in medicated PD patients, the sharp gamma peak present throughout the depth recordings and the lack of bursting firings after levodopa intake have not been reported before. The lack of bursting in SUA, the lack of excessive beta activity and cross frequency coupling between HFOs and lower rhythms further validate the link between bursting firing regime of neurons and pathological oscillatory neural activity in PD-STN. Overall, these observations not only validate the existing literature on the PD electrophysiology in healthy/medicated animal models but also provide insights regarding the underlying electro-pathophysiology of levodopa-induced dyskinesias in PD patients through demonstration of multiscale relationships between single cell firings and field potentials.

Keywords: Parkinson's disease, subthalamic nucleus, levodopa, dyskinesia, single unit activity, local field potentials 


\section{BACKGROUND}

Mapping of the basal ganglia during the implantation of deep brain stimulation (DBS) leads continues to provide researchers insights into the electrophysiology and network dynamics of movement disorders. It is well-known that the structures traversed during intraoperative microelectrode recordings (MER), such as subthalamic nucleus (STN), show distinct patterns both in their spiking (Magariños-Ascone et al., 2000; Rodriguez-Oroz et al., 2001; Starr et al., 2003; Gross et al., 2006; Wong et al., 2009) and oscillatory activity (Wang et al., 2014, 2016; Telkes et al., 2016, 2018) in unmedicated Parkinson's disease (PD) patients. Studies investigating the somatotopic organization of the STN through single cell recordings consistently report that most of the single cell activity related to upper and lower limb movements are located in the dorsolateral region of the STN (Rodriguez-Oroz et al., 2001; Abosch et al., 2002; Theodosopoulos et al., 2003; Weinberger et al., 2006), which consequently have been utilized to target the STN more accurately (Starr et al., 2003). More recent studies have shown that among various spiking profiles that are distributed throughout the STN, bursting and tonic (regular) firings are associated with the symptoms of PD (Sharott et al., 2014; Kaku et al., 2019b). Research in non-human primates also show that the firing type and rate changes in STN neurons can be indicative of healthy, PD or medicated state (Bergman et al., 1994; Soares et al., 2004; Gilmour et al., 2011).

The spatio-spectral characteristics of local field potentials (LFPs) recorded from the same microelectrodes have also been investigated in the territories of STN (Wang et al., 2014; Telkes et al., 2016; van Wijk et al., 2017). Excessive oscillations discovered in the $8-30 \mathrm{~Hz}$ (beta) (Priori et al., 2004; Kühn et al., 2006; Chen et al., 2007; Ray et al., 2008; Eusebio and Brown, 2009) and $200-400 \mathrm{~Hz}$ (high frequency oscillations, HFO) bands, and the cross-frequency coupling (CFC) between these bands (Foffani et al., 2003; Lopez-Azcarate et al., 2010; Özkurt et al., 2011; Hirschmann et al., 2016; van Wijk et al., 2017; Telkes et al., 2018; Ozturk et al., 2019), have been shown to correlate with the motor symptoms (van Wijk et al., 2016; Shreve et al., 2017; Ozturk et al., 2019) and subtypes of the disease (Telkes et al., 2018).

The effect of dopaminergic medication on LFP patterns of the STN has been studied through the postoperative recordings from chronic DBS leads (macroelectrodes). In particular, the excessive beta peak seen in $8-30 \mathrm{~Hz}$ range is suppressed upon dopaminergic medication intake (Brown et al., 2001; Williams et al., 2002; Alonso-Frech et al., 2006; Lopez-Azcarate et al., 2010; Swann et al., 2016; Ozturk et al., 2019). Additionally, the HFO activity seen between 200 and $300 \mathrm{~Hz}$ shifts to 300 and $400 \mathrm{~Hz}$ frequency range and CFC disappears concurrently (Lopez-Azcarate et al., 2010; van Wijk et al., 2016; Ozturk et al., 2019). LFP peaks in $70-90 \mathrm{~Hz}$ range in the STN have also been reported and have been associated with voluntary movement (Cassidy et al., 2002; Cheyne et al., 2008; Thompson et al., 2014) and levodopa-induced dyskinesias (Swann et al., 2016). This modulation of LFPs by PD medication has led to LFP features being used as neuro-biomarkers for closed loop STN stimulation, in which these objective measures facilitate the development of individualized therapies (Hoang et al., 2017; Meidahl et al., 2017; Parastarfeizabadi and Kouzani, 2017).

Although single unit activity (SUA) and LFPs can be recorded simultaneously from microelectrodes, DBS surgeries are typically performed in the unmedicated state. Therefore, the firing profiles of single cells and the spatio-spectral dynamics of microelectrode LFPs en route to and in the territories of STN from medicated subjects remain unexplored. Here we report both SUA and LFP characteristics of a PD patient who underwent bilateral STN DBS implantation while "ON" medication. Our observations from this unique case have the potential to fill the gap between studies from healthy or medicated animal models and PD patients, and provide clues regarding electro-pathophysiology of dyskinesias in medicated patients through single cell and field potential modulations.

\section{METHODS}

\section{Case Presentation}

A 60 year old, right-handed male with idiopathic PD for 16 years was presented for bilateral STN DBS due to bothersome motor fluctuations and severe generalized choreiform and dystonic dyskinesias despite medication optimization. Pre-operatively, his unmedicated Movement Disorders Society Unified Parkinson's Disease Rating Scale (MDS-UPDRS) PartIII score was 53, which reduced to 12 with medication. Though it is requested that patients withhold their dose of dopaminergic agents $12 \mathrm{~h}$ prior to the surgery, the patient took his regular dose of PD medication ( 1 capsule of Amantadine $100 \mathrm{mg}, 3$ capsules of Carbidopa and Levodopa extended release $61.25-245 \mathrm{mg}$ ) in the morning, $2 \mathrm{~h}$ before the surgery. During the recordings, the patient exhibited generalized choreiform dyskinesia. The microelectrode recordings were performed before the placement of the DBS lead, per standard clinical procedure. Starting from left hemisphere, microelectrode recordings were completed in $10 \mathrm{~min}$ and DBS lead was placed to test the patient intraoperatively. Same procedure was followed for the right hemisphere $15 \mathrm{~min}$ after testing the previous side. Compared to baseline, the patient improved clinically by $51 \%$ (pre-op OFF Med: 35, post-op OFF Med/ON Stim: 17) with the electrical stimulation of STN, without any side effects according to the MDS-UPDRS PartIII score (sum of lateral items from both hemispheres: 3.33.8, 3.15-3.17) in 3-month follow up. The patient provided informed consent for these recordings under protocols approved by Institutional Review Boards of the University of Houston and Baylor College of Medicine. Additionally, written informed consent was obtained from the individual for the publication of any potentially identifiable images or data included in this article.

\section{Recordings}

The stereotactic coordinates and trajectories to the STN were identified by fusing preoperative magnetic resonance imaging (MRI) and computerized tomography (CT) scans on a neuronavigational platform (StealthStation, Medtronic, Ireland). This radiographic target was validated using electrophysiological recordings from microelectrodes (NeuroProbe, AlphaOmega, 
Israel) to identify single unit and LFP characteristics of the STN, per standard clinical protocol. The microelectrodes were initially placed at $15 \mathrm{~mm}$ above the target and advanced toward the target in $0.5-1 \mathrm{~mm}$ steps using NeuroOmega drive (AlphaOmega, Israel). At each depth, by using the cannula as reference, at least $20 \mathrm{~s}$ of SUA from the tungsten tip and LFP from the stainlesssteel ring ( $3 \mathrm{~mm}$ above the tip) on the shaft were obtained simultaneously. The dorsal and ventral borders of STN were determined by an experienced neurologist via visual and auditory inspection of SUA activity. The dorsal STN border was identified with a prominent increase in the background activity and this border was used as reference $(0 \mathrm{~mm})$. Depths up to $2.5 \mathrm{~mm}$ ventral to the dorsal border were referred as the "dorsal" half of STN (Telkes et al., 2018; Kaku et al., 2019b). The SUA recordings were obtained with Grapevine bioamplifier (Ripple, UT) at $30 \mathrm{KHz}$ and LFPs were obtained with the same amplifier at $2 \mathrm{KHz}$ sampling frequency and 16 bit $\mathrm{A} / \mathrm{D}$ resolution. The data was stored in a computer hard-drive for offline processing.

\section{Signal Processing}

The recorded signals were processed in MATLAB version R2018a (Mathworks, MA). Following the 2nd order IIR high pass filtering of raw SUA data over $300 \mathrm{~Hz}$, spike analyses were performed automatically by the unsupervised pipeline we proposed recently (Kaku et al., 2019a,b) based on two features, namely Local Variation (LV, Shinomoto et al., 2009) and Bursting Index (BI, Cotterill et al., 2016). Note that BI value 0 indicates no burstiness, whereas 1 is the maximum burstiness. Similarly, LV value approaches to 0 for tonic firings whereas 1 refers to the maximum irregularity. Given the number of isolated neurons, each neuron was visualized manually as well, observing and verifying their classification using raw SUA traces as well as inter-spike interval (ISI) histograms. LFP traces were analyzed in the spectral domain using a modified Welch periodogram. A fast Fourier transform was computed at each depth with a $1 \mathrm{~s}$ Hamming window and $50 \%$ overlap. A median spectrum was calculated from the spectra to eliminate localized artifacts at each depth. Then, individual spectra across depths were combined to generate a 2D depthfrequency map (DFM) representing the depth-varying power spectrum of the LFPs (Telkes et al., 2016). CFC was estimated using a method based on the phase-locking principle (Penny et al., 2008). The comodulograms representing the CFC have amplitude frequency axis from 190 to 410 with $10 \mathrm{~Hz}$ steps and $50 \mathrm{~Hz}$ filter bandwidth and phase frequency axis from 4 to 40 with $1 \mathrm{~Hz}$ steps and $3 \mathrm{~Hz}$ filter bandwidth.

\section{RESULTS}

The raw SUA and LFP data recorded from both hemispheres along the trajectory to the STN are illustrated in Figure 1. Based on the fused intraoperative MRI and intraoperative CT (Figure 1A), the microelectrodes first passed through the anterior thalamus (reticular nucleus) at around $10 \mathrm{~mm}$ above dorsal border. Then, the ventral basal complex of the thalamus was encountered, followed by the zona incerta and STN. The lead trajectories were slightly asymmetric due to anatomical and clinical considerations. The SUA raw traces are given in Figure 1B. Similar to the unmedicated recordings, STN was characterized with increased background activity and single unit firings. It was observed that the single neuronal firing and oscillatory activity spatial profiles were similar between hemispheres. Specifically, the prominent increase in the background activity and firing rate in SUA traces were associated with larger signal amplitude in raw LFP traces (Figure 1D).

Nineteen neurons were isolated in the left STN and seven in the right, totaling 26 neurons in both STNs. The ISI histogram as well as BI and LV values for each tonic and irregularly firing neuron is provided in Figure 1C. The mean firing rate of all neurons was $43 \pm 15 \mathrm{~Hz}$. Among all isolated neurons, $43 \%$ of them demonstrated tonic firing with a mean firing rate of 50 $\pm 16 \mathrm{~Hz}$ whereas $57 \%$ fell into an irregular category with a mean firing rate of $38 \pm 12 \mathrm{~Hz}$. Spatially, the irregular neurons were distributed equally across the dorsal and ventral STN (50\% dorsal and $50 \%$ ventral). Tonic firing patterns, however, were mostly found in the ventral STN (11\% dorsal and $89 \%$ ventral). Based on low BI values $(<0.31)$ and the visual observation of the ISI histograms and the raw traces, no burst-type firings were observed.

The raw traces, depth-frequency maps and the power spectra of LFP at various depths are provided in Figures 1D-F. Throughout the recordings, LFP activity included a dominant low frequency component $(<8 \mathrm{~Hz})$ and a sharp gamma $(70-$ $90 \mathrm{~Hz}$ ) activity. Interestingly, the power of the gamma band was stronger out of STN extending $>10 \mathrm{~mm}$ above it, especially on the right hemisphere, likely due to the larger overlap with the thalamic region on this side (Figure 1A). In both left and right hemisphere spectra, the gamma peak was consistent. Above the STN, this narrow peak was significantly higher than the median power in band with $20 \mathrm{~Hz}$ surrounding it (Wilcoxon rank sum test, $p<0.001$ ). In-STN, the peak was distinguishable but not significantly different than the median (Wilcoxon rank sum test, $p>0.05)$. We also observed another peak with a broad band characteristic between 200 and $400 \mathrm{~Hz}$ (HFO) in the STN. The peak of this band was significantly higher than the median power of the band with $200 \mathrm{~Hz}$ surrounding it (Wilcoxon rank sum test, $p<0.05$ ). The peak in the beta range was also visible but not significantly elevated with respect to its surroundings (Wilcoxon rank sum test, $p>0.05$ ). Despite the presence of strong oscillatory activity in the high frequency range $(200-400 \mathrm{~Hz})$, no cross-frequency coupling in the form of phase-amplitude modulation between beta and HFO bands was detected (Figure 1G).

Following MER, the STN DBS lead was placed and intraoperative test stimulation was performed at constant frequency and pulse width $(130 \mathrm{~Hz}, 60 \mu \mathrm{s})$ with voltages from 1 to $5 \mathrm{~V}$. No side effects were encountered but contralateral dyskinesia improved (Supplementary Video available for the testing of left hemisphere) and recurred with cessation of stimulation.

\section{DISCUSSION}

In this case report, we present unique intraoperative depth recordings of single unit firings and neural oscillations from 
A

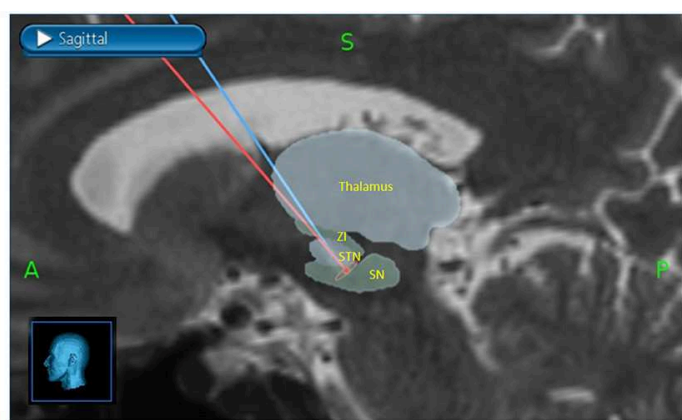

C Tonic Firings
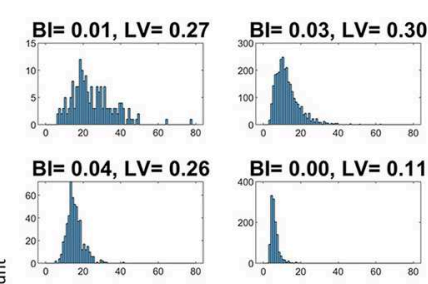

言

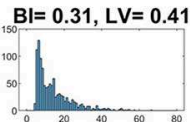

$\mathrm{Bl}=\mathbf{0 . 0 0 , L V = 0 . 1 6}$

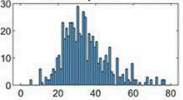

$B=0.00, L V=0.11$

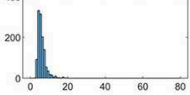

$\mathrm{BI}=0.00, \mathrm{LV}=0.11$

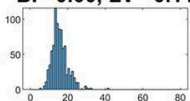

$\mathrm{Bl}=\mathbf{0 . 0 1}, \mathrm{LV}=\mathbf{0 . 3 2}$

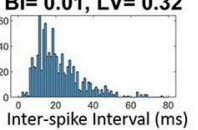

B

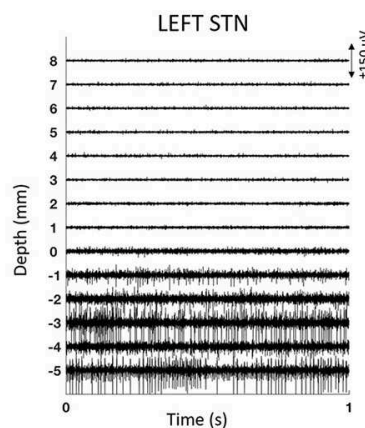

RIGHT STN

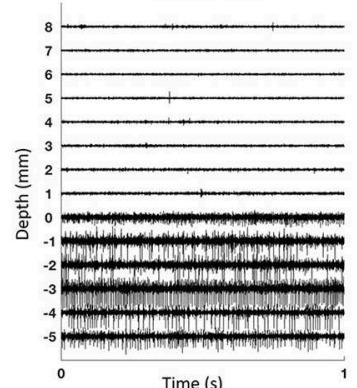

Irregular Firings

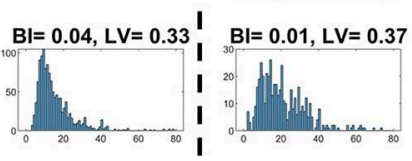

$B I=0.00, L V=0.23, \quad B I=0.02, L V=0.50$

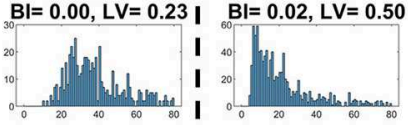

$B|=0.00, L V=0.18 \quad B|=0.02, L V=0.84$

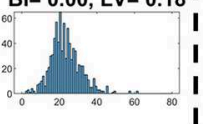

$\mathbf{B l}=\mathbf{0 . 0 1}, \mathbf{L V}=\mathbf{0 . 5 4}$

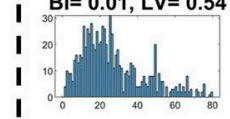

$B I=0.00, L V=0.37$
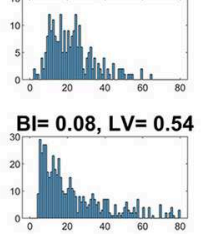

$\mathrm{Bl}=0.01, \mathrm{LV}=0.51$
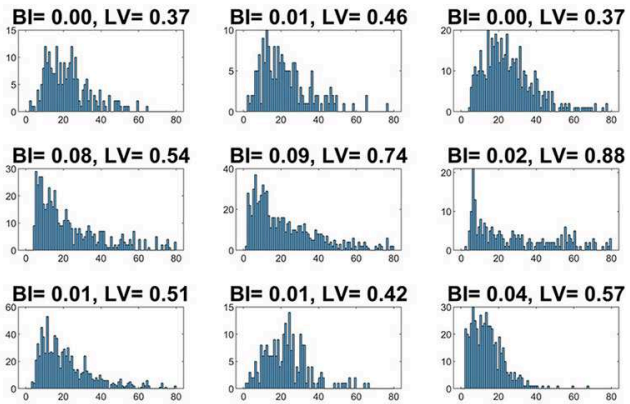

$B=0.09, L V=0.74$

$\mathrm{BI}=0.02, \mathrm{LV}=0.88$

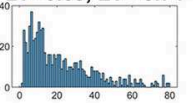

$\mathrm{Bl}=0.01, \mathrm{LV}=0.42$
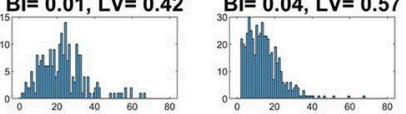

$\mathrm{BI}=0.02, \mathrm{LV}=0.57$

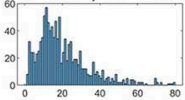

$\mathrm{BI}=\mathbf{0 . 0 5}, \mathrm{LV}=0.98$

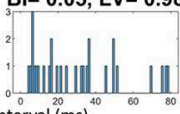

D

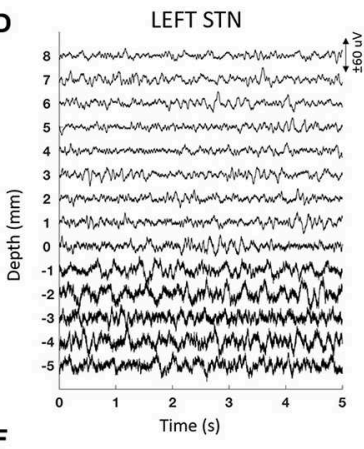

F

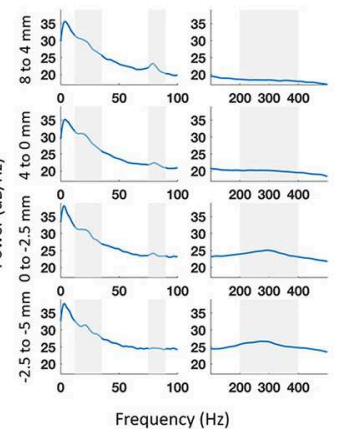

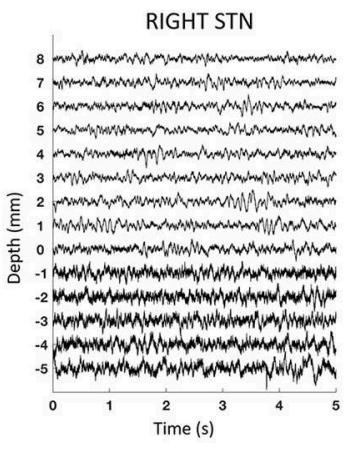

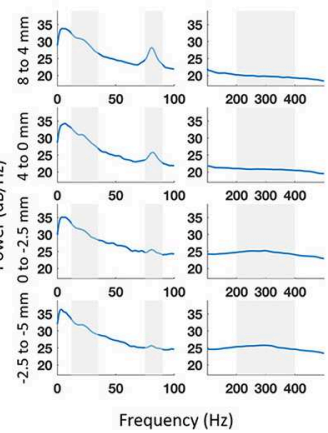

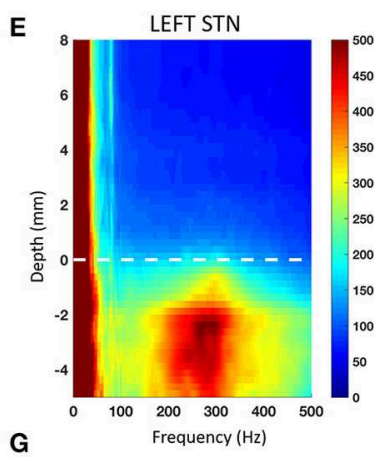
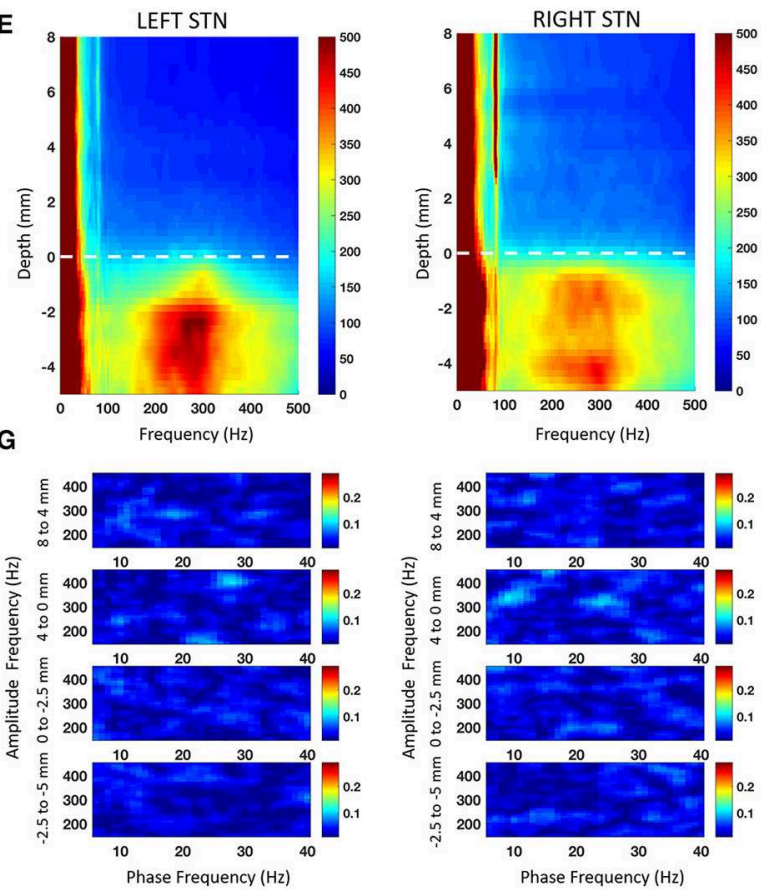

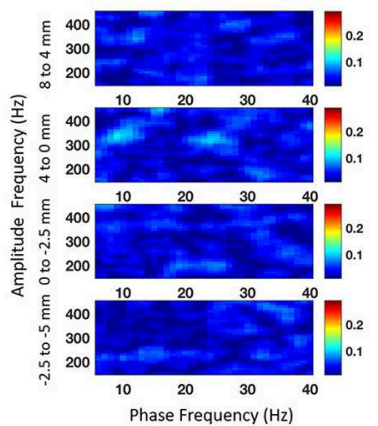

FIGURE 1 | Bilateral SUA and LFP characteristic from intraoperative microelectrode recordings along with the reconstructed trajectories. The depth $0 \mathrm{~mm}$ indicate the dorsal border of STN. (A) Fused intraoperative MRI and intraoperative CT images used for reconstructed microelectrode trajectories en route to STN in StealthStation, superimposed with relevant structures from the Schaltenbrand-Bailey atlas. (B) Sample SUA raw traces with $1 \mathrm{~mm}$ step. (C) ISI histograms of tonic (regular) and irregular firings as well as their BI and LV values. (D) Sample LFP raw traces with $1 \mathrm{~mm}$ step. (E) DFM showing spatio-spectral profile of LFP recordings. Dashed line represents the dorsal border of STN (F) PSD of depth LFPs with 2-4 mm steps. Shaded areas correspond to beta (10-35 Hz), gamma (75-90 Hz), and HFO (200-400 Hz) bands. (G) Comodulograms with 2-4 mm steps, representing coupling in the LFP data with 2-4 mm steps. 
a PD patient who took his full dose of regular medication before the surgery. The patient was a 60 year old male diagnosed with idiopathic PD and was referred to bilateral STNDBS partly due to severe dyskinesias even after optimized medication treatment. The microelectrode recordings starting from $15 \mathrm{~mm}$ above the STN revealed interesting relationships between the SUA and LFP profiles of our medicated patient and healthy/medicated non-human primates and medicated patients.

\section{No Burst Type Firings in the Medicated State}

The identification of different firing types in the STN have been studied previously by others (Bergman et al., 1994; MagariñosAscone et al., 2000; Soares et al., 2004; Weinberger et al., 2006, 2008; Du et al., 2018). We recently contributed to this field with a clustering method to identify bursting, tonic and irregular firing types of STN neurons in an unsupervised fashion (Kaku et al., 2019b). After recording 279 neurons from 20 STNs of 12 unmedicated patients, we reported that the irregular firings dominate the STN firing profile (55\%), followed by bursting (34\%) and then tonic (11\%) firing regimes (Kaku et al., $2019 b)$. These findings on the proportion of bursting firings were also in accordance with studies involving 1-methyl-4phenyl-1,2,3,6-tetrahydropyridine (MPTP) treated non-human primates that report an increase in bursting neuronal activity and instantaneous firing rates after MPTP treatment (Bergman et al., 1994; Soares et al., 2004). Strikingly, in our medicated patient, no burst type firing patterns were observed. Instead, tonic firings increased in proportion without losing their spatial distribution. Out of 26 neurons isolated, 57\% had irregular firing patterns, and $43 \%$ showed tonic firing patterns. The overall mean firing rate $(43 \pm 15 \mathrm{~Hz})$ was comparable to our recent report on 12 unmedicated patients ( $47 \pm 31 \mathrm{~Hz}$ ) (Kaku et al., 2019b). Yet, when we compared the firing rate of patterns separately, we found that while the rate of irregular firings was similar (medicated 38 $\pm 12 \mathrm{~Hz}$ vs. unmedicated $34 \pm 17 \mathrm{~Hz}$ ), the rate of tonic firings was clearly slower in our medicated patient (medicated: $50 \pm$ $16 \mathrm{~Hz}$ vs. unmedicated: $74 \pm 33 \mathrm{~Hz}$ ). On the other hand, reports on man and non-human primates medicated with apomorphine or levodopa show that population firing rates in the STN did not change with drug intake by itself (Lozano et al., 2000; Levy et al., 2001; Gilmour et al., 2011) but did decrease when dyskinesia was present (Mitchell et al., 1992; Lozano et al., 2000), as it did for our medicated patient. Therefore, our observations regarding the firing rate changes could be attributed to not only the therapeutic effect of the drug (MDS-UPDRS part III OFF-ON: 53-12) but also the dyskinetic state it induced. It should be noted that a study involving dyskinetic patients treated with apomorphine reported an increase in "burstiness" in single cell recordings (Levy et al., 2001) which contradicts with our finding of no bursting. They, however, noted that the increase was due to aperiodic bursting. Regardless, this difference could perhaps be due to how levodopa and apomorphine differ in their mechanisms of action (Gilmour et al., 2011). Future studies with more subjects exploring firing patterns before and during dyskinesia periods in subjects administered with levodopa or apomorphine will be able to reach to a more definitive conclusion.

\section{Oscillatory Neural Dynamics in the STN}

Studies investigating STN-LFP oscillations from the DBS lead in medicated patients (Brown et al., 2001; Williams et al., 2002; Alonso-Frech et al., 2006; Lopez-Azcarate et al., 2010; Özkurt et al., 2011; Swann et al., 2016; Ozturk et al., 2019) have shown that dopaminergic medication suppresses the prominent beta peak and $200-300 \mathrm{~Hz}$ HFO in the STN while new peaks may appear in $70-90 \mathrm{~Hz}$ gamma and $300-400 \mathrm{~Hz} \mathrm{HFO}$ range, similar to MPTP treated healthy non-human primates (Escobar et al., 2017). In line with these reports, we observed weak beta activity, a sharp gamma peak at $80 \mathrm{~Hz}$ and a strong broadband HFO centered around $300 \mathrm{~Hz}$ in STN, as shown in Figures 1E,F. Furthermore, we and others have shown that there is coupling between beta and $200-300 \mathrm{~Hz}$ HFO bands in the unmedicated patients that disappears with dopaminergic medication intake (Lopez-Azcarate et al., 2010; Özkurt et al., 2011; van Wijk et al., 2016; Ozturk et al., 2019). Similarly, this coupling was absent in our medicated patient as well. Lack of bursting firings in the SUA and the absence of CFC in the LFP subbands could be an indication of a multiscale relationship between single neuronal and oscillatory activity in PD (Yang et al., 2014; Meidahl et al., 2019).

\section{The Role of Finely Tuned Gamma}

The $70-90 \mathrm{~Hz}$ sharp gamma activity in the STN and in the motor cortex has been labeled as "prokinetic" due to its enhancement with voluntary movement (Cassidy et al., 2002; Cheyne et al., 2008; Thompson et al., 2014). Studies recording together from cortex, pallidum, and STN have also found coherence at the sharp gamma activity between these sites (Cassidy et al., 2002; Williams et al., 2002; Kempf et al., 2009). Unlike previous studies from chronic DBS lead or cortical electrodes, microelectrode recordings shown here sample from various depths above and within STN. Note that in Figures 1E,F, the sharp gamma is not only present within the STN but even up to $10 \mathrm{~mm}$ above the dorsal border, overlapping with thalamus, indicating a broader network synchronization. Furthermore, the sharp gamma activity appears more peaked above the STN in both hemispheres. A previous study recording the same activity from STN and cortex simultaneously reported that it was stronger in cortex (Swann et al., 2016). Another study recording from thalamus and pallidum observed stronger sharp gamma in the thalamus (Kempf et al., 2009). Similarly, the difference in power of the gamma band in our recordings could be stemming from the structures it is recorded from, as we traversed anterior thalamus, zona incerta and arrived at STN. Combined with the cortical recordings, we can speculate that the sharp gamma activity could be present with varying power levels from cortex to subcortical areas. Thus, with its recurring presence in the subcortico-cortical loop, the finely tuned gamma activity might be serving as an "assembling rhythm" between the structures (Buzsáki et al., 2012) and providing a communication channel between them (Fries, 2015). Alternatively, combined with dominant slow oscillations below 
$8 \mathrm{~Hz}$ (Alonso-Frech et al., 2006), the exaggerated gamma activity might also be contributing to the levodopa-induced dyskinesia (LID) generation (Swann et al., 2016). The link between sharp gamma and LID have also been explored at several levels including cortex, striatum and STN of animal models (Halje et al., 2012; Delaville et al., 2015; Belić et al., 2016). Supporting this claim, the patient presented here demonstrated generalized choreiform dyskinesias (Supplementary Video) after levodopa intake which disappeared with STN-DBS (Oyama et al., 2012). It is possible that the -otherwise physiological-gamma rhythm becomes pathological when it is exaggerated due to disrupted "filtering" in the dopamine-overdosed basal ganglia (Weinberger and Dostrovsky, 2011; Swann et al., 2016). Reports of successful thalamotomy/pallidotomy procedures on patients with LID (Narabayashi et al., 1984; Jankovic et al., 1999; Guridi et al., 2012) also support that a circuit level mechanisms is involved in the generation of dyskinesia (Swann et al., 2016; Cenci et al., 2018). Paired with the appropriate processing techniques, sharp gamma oscillations can be used to develop closed-loop technologies to control dyskinesia (Swann et al., 2016).

\section{CONCLUDING REMARKS}

Investigating bilateral microelectrode recordings from above and within STN of a medicated PD patient with dyskinesia for the first time, we have observed that there were no burst type firings unlike unmedicated subjects. Instead, tonic firings with rates slower than unmedicated patients increased in proportion. The oscillatory LFP patterns confirmed previous reports from chronic macroelectrodes in medicated patients as the exaggerated beta and beta-HFO coupling was absent despite the presence of strong HFO band activity. Additionally, sharply tuned gamma oscillations in STN were in accordance with reports from medicated or dyskinetic patients but extension of these oscillations out of STN was not reported before either. Together, these preliminary findings help validate the existing healthy/medicated non-human primate literature on the PD electrophysiology in human subjects and provide insights into the electro-pathophysiology of dyskinesia in the medicated PD patients through increased tonic firing profiles and sharp gamma and HFO activity. Despite lack of concrete evidence, the increase in the proportion of tonic firings in STN and concomitant broadband HFO and sharp gamma oscillations after the medication intake implies that these patterns might be related. Simultaneous recordings from out- and in-STN as well as the cortex in future studies with greater number of subjects might unravel the underlying relationship between tonic firings and these LFP bands in the pathophysiology of PD more clearly.

\section{DATA AVAILABILITY STATEMENT}

The data that support the findings of this study are available on a reasonable request from the corresponding author. The raw data are not publicly available as the data might contain potentially identifying or sensitive information that could compromise the privacy of the research participants.

\section{ETHICS STATEMENT}

The studies involving human participants were reviewed and approved by University of Houston Baylor College of Medicine. The patients/participants provided their written informed consent to participate in this study. Written informed consent was obtained from the individual(s) for the publication of any potentially identifiable images or data included in this article.

\section{AUTHOR CONTRIBUTIONS}

$\mathrm{NI}$ and JJ-S conceived and designed the experiments. MO, HK, and AV performed the experiments. JJ-S and SK performed clinical testing. $\mathrm{MO}, \mathrm{HK}$, and $\mathrm{NI}$ analyzed the data. $\mathrm{MO}$ and $\mathrm{NI}$ wrote the paper. MO, HK, JJ-S, AV, SS, SK, and NI reviewed and revised the manuscript and approved the final manuscript as submitted.

\section{ACKNOWLEDGMENTS}

We would like to thank the patient and his family for their contribution in sharing these unique observations with the community. We also acknowledge the OR supervisor Carolle Daniel and the rest of the operating room personnel at Baylor St Luke's Medical Center for their help in data collection.

\section{SUPPLEMENTARY MATERIAL}

The Supplementary Material for this article can be found online at: https://www.frontiersin.org/articles/10.3389/fnins. 2020.00391/full\#supplementary-material

Supplementary Video | The intraoperative clinical testing for the left STN at constant frequency and pulse width $(130 \mathrm{~Hz}, 60 \mu \mathrm{s})$ with voltages from 1 to $5 \mathrm{~V}$. The medicated patient demonstrated generalized choreiform dyskinesias which disappeared after STN-DBS was turned ON. No side effects were observed.

\section{REFERENCES}

Abosch, A., Hutchison, W. D., Saint-Cyr, J. A., Dostrovsky, J. O., and Lozano, A. M. (2002). Movement-related neurons of the subthalamic nucleus in patients with Parkinson disease. J. Neurosurg. 97, 1167-1172. doi: 10.3171/jns.2002.97.5.1167 Alonso-Frech, F., Zamarbide, I., Alegre, M., Rodríguez-Oroz, M. C., Guridi, J., Manrique, M., et al. (2006). Slow oscillatory activity and levodopainduced dyskinesias in Parkinson's disease. Brain 129, 1748-1757. doi: 10.1093/brain/awl103

Belić, J. J., Halje, P., Richter, U., Petersson, P., and Hellgren Kotaleski, J. (2016). Untangling cortico-striatal connectivity and cross-frequency coupling in L-DOPA-induced dyskinesia. Front. Syst. Neurosci. 10:26. doi: 10.3389/fnsys.2016.00026

Bergman, H., Wichmann, T., Karmon, B., and DeLong, M. R. (1994). The primate subthalamic nucleus. II. neuronal activity in the MPTP model of parkinsonism. J. Neurophysiol. 72, 507-520. doi: 10.1152/jn.1994.72.2.507

Brown, P., Oliviero, A., Mazzone, P., Insola, A., Tonali, P., and Di Lazzaro, V. (2001). Dopamine dependency of oscillations between subthalamic 
nucleus and pallidum in Parkinson's disease. J. Neurosci. 21, 1033-1038. doi: 10.1523/JNEUROSCI.21-03-01033.2001

Buzsáki, G., Anastassiou, C. A., and Koch, C. (2012). The origin of extracellular fields and currents - EEG, ECoG, LFP and spikes. Nat. Rev. Neurosci. 13, 407-420. doi: 10.1038/nrn3241

Cassidy, M., Mazzone, P., Oliviero, A., Insola, A., Tonali, P., Di Lazzaro, V., et al. (2002). Movement-related changes in synchronization in the human basal ganglia. Brain 125, 1235-1246. doi: 10.1093/brain/awf135

Cenci, M. A., Jörntell, H., and Petersson, P. (2018). On the neuronal circuitry mediating l-DOPA-induced dyskinesia. J. Neural. Transm. 125, 1157-1169. doi: 10.1007/s00702-018-1886-0

Chen, C. C., Litvak, V., Gilbertson, T., Kühn, A., Lu, C. S., Lee, S. T., et al. (2007). Excessive synchronization of basal ganglia neurons at $20 \mathrm{~Hz}$ slows movement in Parkinson's disease. Exp. Neurol. 205, 214-221. doi: 10.1016/j.expneurol.2007.01.027

Cheyne, D., Bells, S., Ferrari, P., Gaetz, W., and Bostan, A. C. (2008). Selfpaced movements induce high-frequency gamma oscillations in primary motor cortex. Neuroimage 42, 332-342. doi: 10.1016/j.neuroimage.2008.04.178

Cotterill, E., Charlesworth, P., Thomas, C. W., Paulsen, O., and Eglen, S. J. (2016). A comparison of computational methods for detecting bursts in neuronal spike trains and their application to human stem cell-derived neuronal networks. J. Neurophysiol. 116, 306-321. doi: 10.1152/jn.00093.2016

Delaville, C., McCoy, A. J., Gerber, C. M., Cruz, A. V., and Walters, J. R. (2015). Subthalamic nucleus activity in the awake hemiparkinsonian rat: relationships with motor and cognitive networks. J. Neurosci. 35, 6918-6930. doi: 10.1523/JNEUROSCI.0587-15.2015

Du, G., Zhuang, P., Hallett, M., Zhang, Y.-Q., Li, J.-Y., and Li, Y.-J. (2018). Properties of oscillatory neuronal activity in the basal ganglia and thalamus in patients with Parkinson's disease. Transl. Neurodegener. 7:17. doi: 10.1186/s40035-018-0123-y

Escobar, D., Johnson, L. A., Nebeck, S. D., Zhang, J., Johnson, M. D., Baker, K. B., et al. (2017). Parkinsonism and Vigilance: alteration in neural oscillatory activity and phase-amplitude coupling in the basal ganglia and motor cortex. J. Neurophysiol. 118, 2654-2669. doi: 10.1152/jn.00388.2017

Eusebio, A., and Brown, P. (2009). Synchronisation in the beta frequency-band the bad boy of parkinsonism or an innocent bystander? Exp. Neurol. 217, 1-3. doi: 10.1016/j.expneurol.2009.02.003

Foffani, G., Priori, A., Egidi, M., Rampini, P., Tamma, F., Caputo, E., et al. (2003). 300-Hz subthalamic oscillations in Parkinson's disease. Brain 126, 2153-2163. doi: 10.1093/brain/awg229

Fries, P. (2015). Rhythms for cognition: communication through coherence. Neuron 88, 220-235. doi: 10.1016/j.neuron.2015.09.034

Gilmour, T. P., Lieu, C. A., Nolt, M. J., Piallat, B., Deogaonkar, M., and Subramanian, T. (2011). The effects of chronic levodopa treatments on the neuronal firing properties of the subthalamic nucleus and substantia nigra reticulata in hemiparkinsonian rhesus monkeys. Exp. Neurol. 228, 53-58. doi: 10.1016/j.expneurol.2010.12.001

Gross, R. E., Krack, P., Rodriguez-Oroz, M. C., Rezai, A. R., and Benabid, A.L. (2006). Electrophysiological mapping for the implantation of deep brain stimulators for Parkinson's disease and tremor. Mov. Disord. 21, S259-S283. doi: $10.1002 / \mathrm{mds} .20960$

Guridi, J., González-Redondo, R., and Obeso, J. A. (2012). Clinical features, pathophysiology, and treatment of levodopa-induced dyskinesias in parkinson's disease. Parkinsons Dis. 2012, 1-15. doi: 10.1155/2012/943159

Halje, P., Tamte, M., Richter, U., Mohammed, M., Cenci, M. A., and Petersson, P. (2012). Levodopa-induced dyskinesia is strongly associated with resonant cortical oscillations. J. Neurosci. 32, 16541-16551. doi: 10.1523/JNEUROSCI.3047-12.2012

Hirschmann, J., Butz, M., Hartmann, C. J., Hoogenboom, N., and Özkurt, T. E., Vesper, J., et al. (2016). Parkinsonian rest tremor is associated with modulations of subthalamic high-frequency oscillations. Mov. Disord. 31, 1551-1559. doi: 10.1002/mds.26663

Hoang, K. B., Cassar, I. R., Grill, W. M., and Turner, D. A. (2017). Biomarkers and stimulation algorithms for adaptive brain stimulation. Front. Neurosci. 11:564. doi: 10.3389/fnins.2017.00564

Jankovic, J., Lai, E., Ben-Arie, L., Krauss, J. K., and Grossman, R. (1999). Levodopa-induced dyskinesias treated by pallidotomy. J. Neurol. Sci. 167, 62-67. doi: 10.1016/S0022-510X(99)00141-0
Kaku, H., Ozturk, M., Viswanathan, A., Jimenez-Shahed, J., Sheth, S., and Ince, N. F. (2019a). "Grouping neuronal spiking patterns in the subthalamic nucleus of parkinsonian patients," in 2019 41st Annual International Conference of the IEEE Engineering in Medicine and Biology Society (EMBC) (Berlin: IEEE), 4221-4224.

Kaku, H., Ozturk, M., Viswanathan, A., Shahed, J., Sheth, S., Kumar, S., et al. (2019b). Unsupervised clustering reveals spatially varying single neuronal firing patterns in the subthalamic nucleus of patients with Parkinson's disease. Clin. Park Relat. Disord. 3:100032. doi: 10.1101/863464

Kempf, F., Brücke, C., Salih, F., Trottenberg, T., Kupsch, A., Schneider, G. H., et al. (2009). Gamma activity and reactivity in human thalamic local field potentials. Eur. J. Neurosci. 29, 943-953. doi: 10.1111/j.1460-9568.2009.06655.x

Kühn, A. A., Kupsch, A., Schneider, G. H., and Brown, P. (2006). Reduction in subthalamic $8-35 \mathrm{~Hz}$ oscillatory activity correlates with clinical improvement in Parkinson's disease. Eur. J. Neurosci. 23, 1956-1960. doi: 10.1111/j.1460-9568.2006.04717.x

Levy, R., Dostrovsky, J. O., Lang, A. E., Sime, E., Hutchison, W. D., and Lozano, A. M. (2001). Effects of apomorphine on subthalamic nucleus and globus pallidus internus neurons in patients with Parkinson's disease. J. Neurophysiol. 86, 249-260. doi: 10.1152/jn.2001.86.1.249

Lopez-Azcarate, J., Tainta, M., Rodriguez-Oroz, M. C., Valencia, M., Gonzalez, R., Guridi, J., et al. (2010). Coupling between beta and high-frequency activity in the human subthalamic nucleus may be a pathophysiological mechanism in Parkinson's disease. J. Neurosci. 30, 6667-6677. doi: 10.1523/JNEUROSCI.5459-09.2010

Lozano, A., Lang, A. E., Levy, R., Hutchison, W., and Dostrovsky, J. (2000). Neural recordings in Parkinson's disease patients with dyskinesias induced by apomorphine. Ann. Neurol. 47, S141-S146.

Magariños-Ascone, C. M., Figueiras-Mendez, R., Riva-Meana, C., and CórdobaFernández, A. (2000). Subthalamic neuron activity related to tremor and movement in Parkinson's disease. Eur. J. Neurosci. 12, 2597-2607. doi: 10.1046/j.1460-9568.2000.00127.x

Meidahl, A. C., Moll, C. K. E., van Wijk, B. C. M., Gulberti, A., Tinkhauser, G., Westphal, M., et al. (2019). Synchronised spiking activity underlies phase amplitude coupling in the subthalamic nucleus of Parkinson's disease patients. Neurobiol. Dis. 127, 101-113. doi: 10.1016/j.nbd.2019.02.005

Meidahl, A. C., Tinkhauser, G., Herz, D. M., Cagnan, H., Debarros, J., and Brown, P. (2017). Adaptive deep brain stimulation for movement disorders: the long road to clinical therapy. Mov. Disord. 32, 810-819. doi: 10.1002/mds. 27022

Mitchell, I. J., Crossman, A. R., Liminga, U., Andren, P., and Gunne, L. M. (1992). Regional changes in 2-deoxyglucose uptake associated with neurolepticinduced tardive dyskinesia in the Cebus monkey. Mov. Disord. 7, 32-37. doi: $10.1002 /$ mds. 870070106

Narabayashi, H., Yokochi, F., and Nakajima, Y. (1984). Levodopa-induced dyskinesia and thalamotomy. J. Neurol. Neurosurg. Psychiatry 47, 831-839. doi: $10.1136 /$ jnnp. 47.8 .831

Oyama, G., Foote, K. D., Jacobson, C. E., Velez-Lago, F., Go, C., Limotai, N., et al. (2012). GPi and STN deep brain stimulation can suppress dyskinesia in Parkinson's disease. Park. Relat. Disord. 18, 814-818. doi: 10.1016/j.parkreldis.2012.03.022

Özkurt, T. E., Butz, M., Homburger, M., Elben, S., and Vesper, J., Wojtecki, L., et al. (2011). High frequency oscillations in the subthalamic nucleus: a neurophysiological marker of the motor state in Parkinson's disease. Exp. Neurol. 229, 324-331. doi: 10.1016/j.expneurol.2011.02.015

Ozturk, M., Abosch, A., Francis, D., Wu, J., Jimenez-Shahed, J., and Ince, N. F. (2019). Distinct subthalamic coupling in the ON state describes motor performance in Parkinson's disease. Mov. Disord. doi: 10.1002/mds.27800

Parastarfeizabadi, M., and Kouzani, A. Z. (2017). Advances in closedloop deep brain stimulation devices. J. Neuroeng. Rehabil. 14:79. doi: 10.1186/s12984-017-0295-1

Penny, W. D., Duzel, E., Miller, K. J., and Ojemann, J. G. (2008). Testing for nested oscillation. J. Neurosci. Methods 174, 50-61. doi: 10.1016/j.jneumeth.2008.06.035

Priori, A., Foffani, G., Pesenti, A., Tamma, F., Bianchi, A. M., Pellegrini, M., et al. (2004). Rhythm-specific pharmacological modulation of subthalamic activity in Parkinson's disease. Exp. Neurol. 189, 369-379. doi: 10.1016/j.expneurol.2004.06.001 
Ray, N. J., Jenkinson, N., Wang, S., Holland, P., Brittain, J. S., Joint, C., et al. (2008). Local field potential beta activity in the subthalamic nucleus of patients with Parkinson's disease is associated with improvements in bradykinesia after dopamine and deep brain stimulation. Exp. Neurol. 213, 108-113. doi: 10.1016/j.expneurol.2008.05.008

Rodriguez-Oroz, M. C., Rodriguez, M., Guridi, J., Mewes, K., Chockkman, V., Vitek, J., et al. (2001). The subthalamic nucleus in Parkinson's disease: somatotopic organization and physiological characteristics. Brain 124, 1777-1790. doi: 10.1093/brain/124.9.1777

Sharott, A., Gulberti, A., Zittel, S., Tudor Jones, A. A., Fickel, U., Munchau, A., et al. (2014). Activity parameters of subthalamic nucleus neurons selectively predict motor symptom severity in parkinson's disease. J. Neurosci. 34, 6273-6285. doi: 10.1523/JNEUROSCI.1803-13.2014

Shinomoto, S., Kim, H., Shimokawa, T., Matsuno, N., Funahashi, S., Shima, K., et al. (2009). Relating neuronal firing patterns to functional differentiation of cerebral cortex. PLoS Comput. Biol. 5:100433. doi: 10.1371/journal.pcbi.1000433

Shreve, L. A., Velisar, A., Malekmohammadi, M., Koop, M. M., Trager, M., Quinn, E. J., et al. (2017). Subthalamic oscillations and phase amplitude coupling are greater in the more affected hemisphere in Parkinson's disease. Clin. Neurophysiol. 128, 128-137. doi: 10.1016/j.clinph.2016.10.095

Soares, J., Kliem, M. A., Betarbet, R., Greenamyre, J. T., Yamamoto, B., and Wichmann, T. (2004). Role of external pallidal segment in primate parkinsonism: comparison of the effects of 1-methyl-4-phenyl-1,2,3,6tetrahydropyridine-induced parkinsonism and lesions of the external pallidal segment. J. Neurosci. 24, 6417-6426. doi: 10.1523/JNEUROSCI.0836-04.2004

Starr, P. A., Theodosopoulos, P. V., and Turner, R. (2003). Surgery of the subthalamic nucleus: use of movement-related neuronal activity for surgical navigation. Neurosurgery 53, 1146-9. doi: 10.1227/01.NEU.0000088803.79153.05

Swann, N. C., de Hemptinne, C., Miocinovic, S., Qasim, S., Wang, S. S., Ziman, N., et al. (2016). Gamma oscillations in the hyperkinetic state detected with chronic human brain recordings in parkinson's disease. J. Neurosci. 36, 6445-6458. doi: 10.1523/JNEUROSCI.1128-16.2016

Telkes, I., Jimenez-Shahed, J., Viswanathan, A., Abosch, A., and Ince, N. F. (2016). Prediction of STN-DBS electrode implantation track in Parkinson's disease by using local field potentials. Front. Neurosci. 10:198. doi: 10.3389/fnins.2016.00198

Telkes, I., Viswanathan, A., Jimenez-Shahed, J., Abosch, A., Ozturk, M., Gupte, A., et al. (2018). Local field potentials of subthalamic nucleus contain electrophysiological footprints of motor subtypes of Parkinson's disease. Proc. Natl. Acad. Sci. U.S.A. 115, E8567-E8576. doi: 10.1073/pnas.1810589115

Theodosopoulos, P. V., Marks, W. J., Christine, C., and Starr, P. A. (2003). Locations of movement-related cells in the human subthalamic nucleus in Parkinson's disease. Mov. Disord. 18, 791-798. doi: 10.1002/mds. 10446

Thompson, J. A., Lanctin, D., Ince, N. F., and Abosch, A. (2014). Clinical implications of local field potentials for understanding and treating movement disorders. Stereotact. Funct. Neurosurg. 92, 251-263. doi: 10.1159/000364913

van Wijk, B. C. M., Beudel, M., Jha, A., Oswal, A., Foltynie, T., Hariz, M. I., et al. (2016). Subthalamic nucleus phase-amplitude coupling correlates with motor impairment in Parkinson's disease. Clin. Neurophysiol. 127, 2010-2019. doi: 10.1016/j.clinph.2016.01.015

van Wijk, B. C. M., Pogosyan, A., Hariz, M. I., Akram, H., Foltynie, T., Limousin, P., et al. (2017). Localization of beta and high-frequency oscillations within the subthalamic nucleus region. NeuroImage Clin. 16, 175-183. doi: 10.1016/j.nicl.2017.07.018

Wang, D. D., de Hemptinne, C., Miocinovic, S., Qasim, S. E., Miller, A. M., Ostrem, J. L., et al. (2016). Subthalamic local field potentials in Parkinson's disease and isolated dystonia: An evaluation of potential biomarkers. Neurobiol. Dis. 89, 213-222. doi: 10.1016/j.nbd.2016.02.015

Wang, J., Hirschmann, J., Elben, S., Hartmann, C. J., Vesper, J., Wojtecki, L., et al. (2014). High-frequency oscillations in Parkinson's disease: spatial distribution and clinical relevance. Mov. Disord. 29, 1265-1272. doi: 10.1002/mds.25962

Weinberger, M., and Dostrovsky, J. O. (2011). A basis for the pathological oscillations in basal ganglia: the crucial role of dopamine. Neuroreport 22, 151-156. doi: 10.1097/WNR.0b013e32 8342 ba 50

Weinberger, M., Hutchison, W. D., Lozano, A. M., Hodaie, M., and Dostrovsky, J. O. (2008). Increased gamma oscillatory activity in the subthalamic nucleus during tremor in parkinson's disease patients. J. Neurophysiol. 101, 789-802. doi: 10.1152/jn.90837.2008

Weinberger, M., Mahant, N., Hutchison, W. D., Lozano, A. M., Moro, E., Hodaie, M., et al. (2006). Beta oscillatory activity in the subthalamic nucleus and its relation to dopaminergic response in parkinson's disease. J. Neurophysiol. 96, 3248-3256. doi: 10.1152/jn.00697.2006

Williams, D., Tijssen, M., Van Bruggen, G., Bosch, A., Insola, A., Di Lazzaro, V., et al. (2002). Dopamine-dependent changes in the functional connectivity between basal ganglia and cerebral cortex in humans. Brain 125, 1558-1569. doi: 10.1093/brain/awf156

Wong, S., Baltuch, G. H., Jaggi, J. L., and Danish, S. F. (2009). Functional localization and visualization of the subthalamic nucleus from microelectrode recordings acquired during DBS surgery with unsupervised machine learning. J. Neural. Eng. 6:026006. doi: 10.1088/1741-2560/6/2/ 026006

Yang, A. I., Vanegas, N., Lungu, C., and Zaghloul, K. A. (2014). Betacoupled high-frequency activity and beta-locked neuronal spiking in the subthalamic nucleus of parkinson's disease. J. Neurosci. 34, 12816-12827. doi: 10.1523/JNEUROSCI.1895-14.2014

Conflict of Interest: The authors declare that the research was conducted in the absence of any commercial or financial relationships that could be construed as a potential conflict of interest.

Copyright (C) 2020 Ozturk, Kaku, Jimenez-Shahed, Viswanathan, Sheth, Kumar and Ince. This is an open-access article distributed under the terms of the Creative Commons Attribution License (CC BY). The use, distribution or reproduction in other forums is permitted, provided the original author(s) and the copyright owner(s) are credited and that the original publication in this journal is cited, in accordance with accepted academic practice. No use, distribution or reproduction is permitted which does not comply with these terms. 